\title{
Vascular Aging and Arterial Stiffness in Older Adults
}

\author{
Erika Maria Gonçalves Campana ${ }^{1,2}$ and Sayuri Inuzuka ${ }^{2,3}$ (을 \\ Universidade do Estado do Rio de Janeiro (UERJ), ${ }^{\prime}$ Rio de Janeiro, RJ - Brazil. \\ Universidade de Nova Iguaçu, ${ }^{2}$ Rio de Janeiro, $R J$ - Brazil. \\ Universidade Federal de Goiás (UFG), ${ }^{3}$ Goiás, GO - Brazil. \\ Editorial related to the article: Determinants of Arterial Stiffness and Vascular Aging in the Older Adult
}

Deaths from cardiovascular disease, according to the $\mathrm{WHO}$, are projected to reach around 14 million by 2030. The global population is aging at an accelerated rate and the prevalence of arterial hypertension $(\mathrm{AH})$ increases with advancing age. ${ }^{1}$ Aging is the common denominator in several cardiovascular diseases. Arterial stiffness and increased pulse wave velocity (PWV), as well as central systolic pressure are major predictors of cardiovascular events. (Figure 1) ${ }^{2}$

Arterial stiffness reflects the true arterial wall damage of risk factors, and it increases with aging. On the other hand, mean blood pressure (MBP), glycemia and lipids are circulating markers whose values fluctuate along the follow-up of patients. Thus, measuring circulating biomarkers at a certain time may give only a snapshot and not the whole history of arterial wall damage. As shown in Figure 2, the gray zone before the time at measurement, when the risk assessment of a hypothetical patient is done, represents a period of life when circulating biomarkers may present altered values and, consequently, aggression to the vascular wall, interchanged with periods of good control of circulating biomarkers. This indicates that, most often, the physician does not know the amount of exposure to $\mathrm{CV}$ risk factors that a patient presents throughout life. Because risk scores perform a cross-sectional analysis of a single moment, they may not be able to quantify the overall cardiovascular and residual risks of each patient. However, the evaluation of arterial stiffness expresses the sum of all the aggressions caused by

\section{Keywords}

Cardiovascular Diseases/mortality; Hypertension; Vascular stiffness; Risk factors; Biomarkers; Blood Pressure; Aged; Frailty; Pulse Wave Analyses. circulating markers on the arterial wall over time and, for this reason, it expresses more accurately the future risk of a CV event or death. ${ }^{2}$ (Figure 2)

Further assessment and stratification of risk, in addition to the biomarkers already established, such as blood pressure (BP), blood glucose, cholesterol and carotid intima-media thickness, must provide a better and cost-effective risk prediction. Therefore, a study investigated whether a $1 \mathrm{~m} / \mathrm{s}$ increase in $\mathrm{PWV}$ was associated with a $7 \%$ increased risk of a cardiovascular event for a 60 year old man. ${ }^{3}$

In addition, the altered behavior of central hemodynamic parameters, such as central systolic blood pressure (cSBP) and the (heart rate-corrected) augmentation index (AI), is influenced by arterial stiffness and plays a crucial role in the interface between the traditional cardiovascular continuum vascular and aging continuum, which represents the current view of the pathophysiology of cardiovascular diseases. ${ }^{4-6}$

In addition to cardiovascular impairment, another concern related to aging is cognition. A review showed that arterial stiffness severity was positively correlated with cognitive impairment. The mechanisms may be associated with greater arterial pulsatility, damaging the cerebral microcirculation, which causes various phenomena associated with cerebral small vessel diseases. It may also be associated with reductions in white matter and gray matter integrity, medial temporal lobe atrophy and $\mathrm{A} \beta$ protein deposition. ${ }^{7}$

Emerging evidence suggests that frailty is a risk factor for CVD. Individuals are considered frail if they present at least three out of these five items: unintentional weight loss of $\geq 10 \mathrm{lbs}$ within a year, self-reported exhaustion, weakness as measured by grip strength, slow walking speed, and decreased physical activity. High frailty levels are associated with increased stiffness. ${ }^{8}$

Mailing Address: Erika Maria Gonçalves Campana

Rua São Januário, 159 / 1103 B. Postal Code: 24130386, Fonseca - Niterói, RJ - Brazil.

E-mail: erikamaria@cardiol.br, campanaemg@predialnet.com.br

DOI: https://doi.org/10.36660/ijcs.20200132 


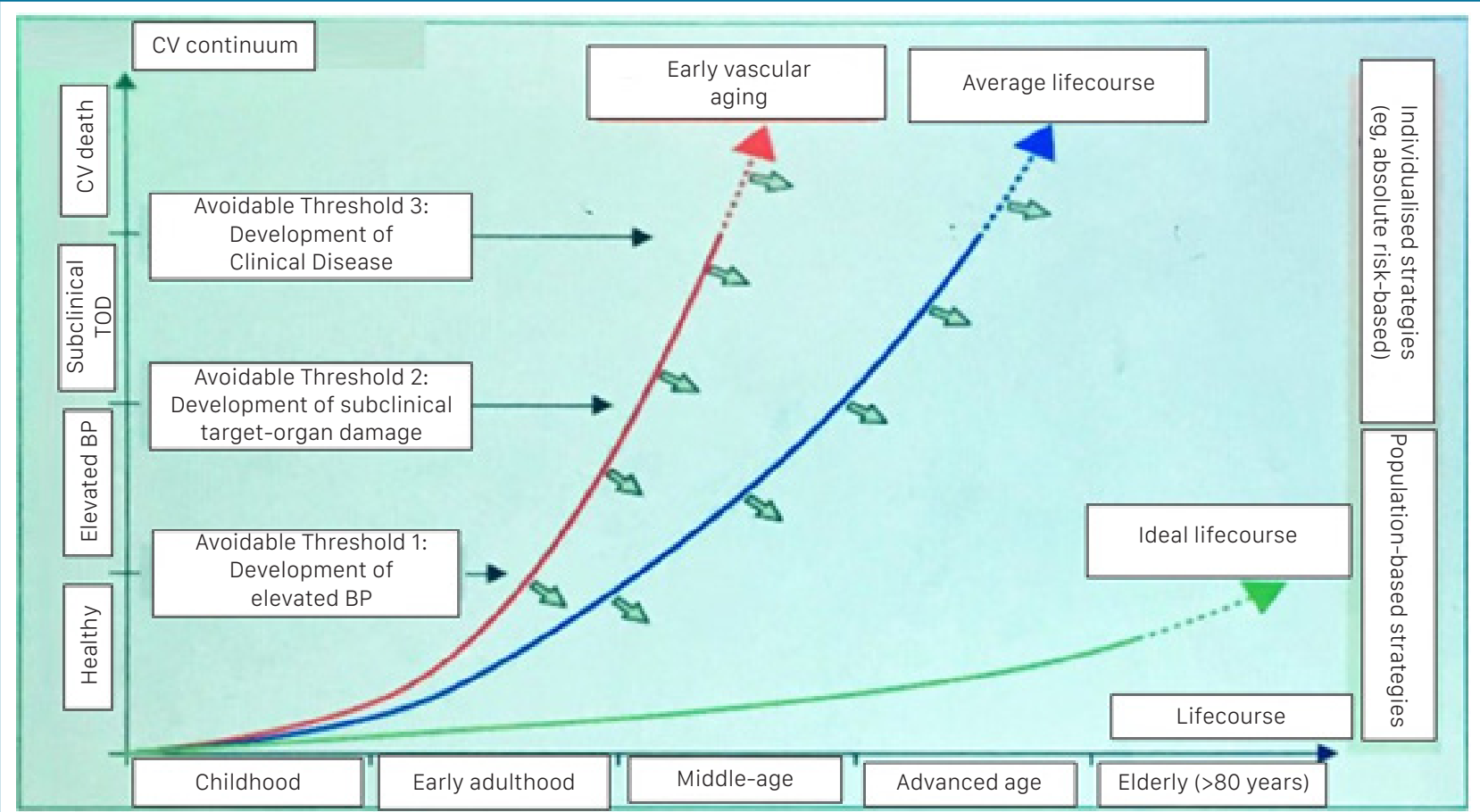

Figure 1 - Ideal lifecourse and vascular aging (Adapted from Nilsson et al Early Vascular Aging and Prevention)[2] CV: cardiovascular; TOD: target organ damage; BP: blood pressure.

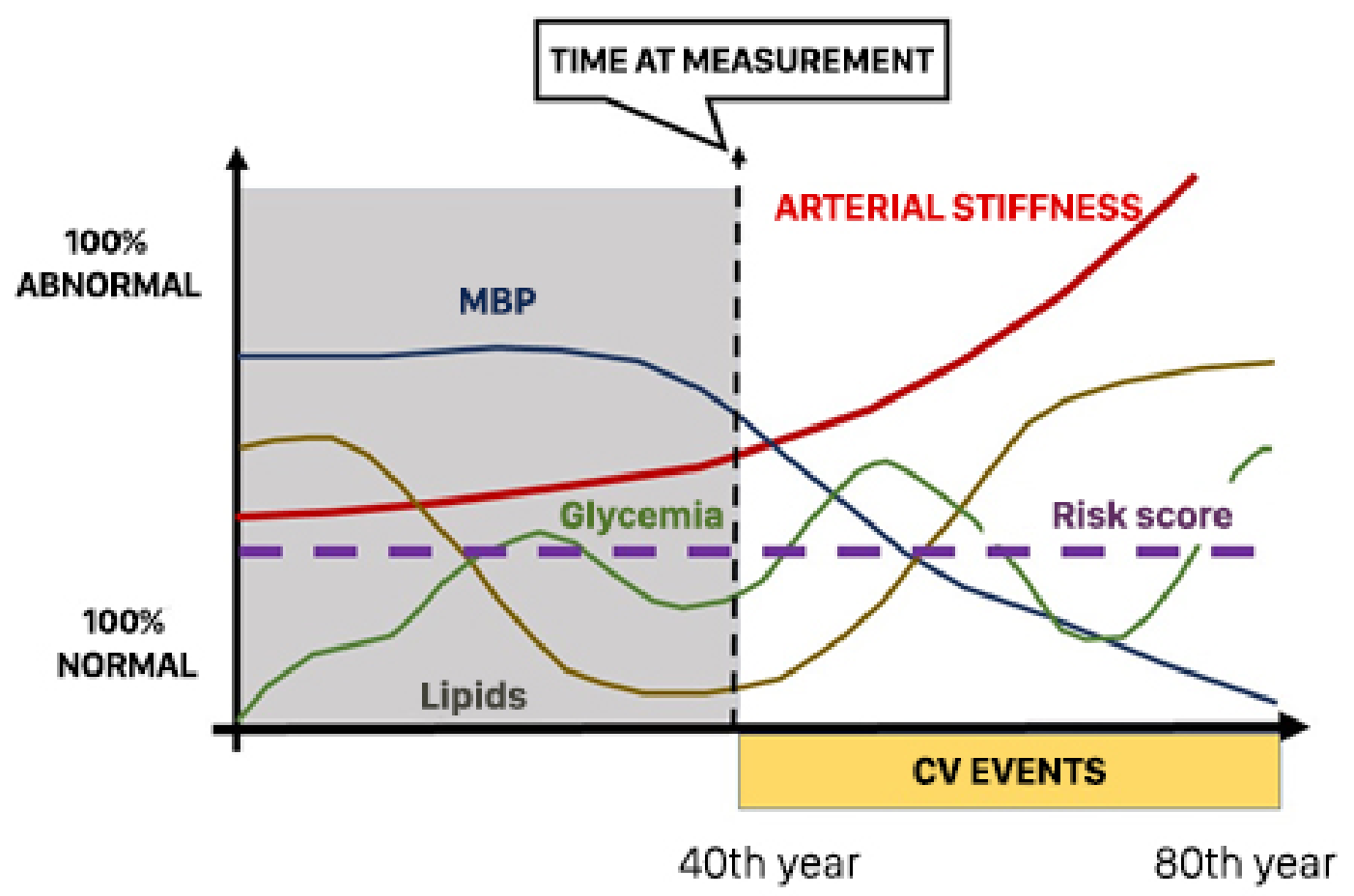

Figure 2 - Vascular Aging (Adapted from Nilsson et al Early Vascular Aging and Prevention). Mean BP (MBP); Cardiovascular (CV) events[2]. 
The article published in this issue was a crosssectional, observational study of participants enrolled in the AGA@4life project, developed to evaluate the effects of several interventions (psychological, physical and nutritional therapy). The aim of the preliminary analysis was to identify the main determinants of arterial stiffness in the very old, identifying the factors that may accelerate arterial ageing and potential routes for preventive actions targeting the maintenance of vascular health. The study enrolled 54 elderly aged between 65 and 94 years from a day care center, in Vilarinho, Portugal. ${ }^{9}$

Arterial stiffness was obtained by an oscillometric method that uses the Mobil-O-Graph, a method validated in previous studies. Hypertension was observed in the large majority of the participants $(80 \%)$ which, in its isolated form, is an important cardiovascular risk factor; however, only $64 \%$ were under treatment. Aging was seen as a determinant factor for PWV and blood pressure, and there was an intrinsic relationship between PWV and blood pressure. The study also showed an association between PWV and renal function, as well as frailty.

Most of the knowledge on accelerated vascular aging has been acquired from the general population, not including the most advanced age group. Therefore, researches on arterial stiffness in the elderly, such as the one carried out by the AGA@4life project, ${ }^{9}$ are important to develop adequate and useful intervention programs for a better cardiovascular protection of this group.

\section{References}

1. World Health Organization. (WHO). A global brief on hypertension: silent killer, global public health crisis. Geneva; 2013.

2. Nilsson PM, Boutouyrie P, Laurent S. Vascular aging: A tale of EVA and ADAM in cardiovascular risk assessment and prevention. Hypertension. 2009;54(1):3-10

3. Ben-Shlomo Y,Spears M, Boustred C, May M, Anderson SG, Benjamin E, et al. Aortic pulse wave velocity improves cardiovascular event prediction: an individual participant meta-analysis of prospective observational data from 17,635 subjects. J Am Coll Cardiol. 2014;63(7):636-46.

4. Mikael LR, Paiva AM, Gomes MM, Euzebio MB, Jardim PCB, Vitorino PVO, et al. Vascular Aging and Arterial Stiffness. Arq Bras Cardiol.2017;109(3):253-8.

5. Roman MJ, Devereux R, Kiezer JR, Lee ET, Gallowby JM, Howard BV. Central pressure more strongly relates to vascular disease

and outcomthan does brachial pressure: the Strong Heart Study Hypertension. 2007;50(1):197-203.

6. Dzau VJ, Antman EM, Black HR, Hayes DL, Manson JE, Plutzky J, Popma JJ, et al. The cardiovascular disease continuum validated: clinical evidence of improved patient outcomes: part I: Pathophysiology and clinical trial evidence (risk factors through stable coronary artery disease). Circulation.2006;114(25):2850-70.

7. Li X, Liu P, Ren Y, An J, Dong Y. Arterial stiffness and cognitive impairment. J Neurol Sci. 2017 Sep 15; 380:1-10.

8. Orkaby A, Lunetta KL, SunF, Driver JA, Benjamin EJ,Hamburg NM, etal. CrossSectional Association of Frailty and Arterial Stiffness in Community-Dwelling Older Adults: The Framingham Heart Study. J Gerontol.2019. 74(3):373-9.

9. Pereira T, Costa T. Determinants of Arterial Stiffness and Vascular Aging in the Older Adult. Int J Cardiovasc Sci. 2020; 33(4):349-356. DOI: https:// doi.org/10.36660/ijcs.20190068 